\title{
Research on the Modern Psychological Health Problems of the Floating Population Development Trend under the Urbanization Process
}

\author{
Dengguo Zhang \\ Department of Management Science, \\ Shandong Provincial Party School of the CPC.
}

\begin{abstract}
In this paper, we conduct research on modern psychological health problems of floating population development trend under the urbanization process. Psychological health problems in our country and its influence factors have been seriously, and the relationship between self-concept and mental health of empirical research is still rare, less accumulation. In addition, many western research is mostly discussed the overall self-concept influence on the mental health, and less consider the self-0concept specific aspects affect individual health or social behavior. Under this background, we propose the novel perspective on the psychological health problems of floating population that will enhance the contemporary research on the related topics.
\end{abstract}

Keywords- Psychological Health, Floating Population, Trend, Urbanization Process, Modern.

\section{Introduction}

By the area and population urbanization is a reflection of the change which is a continuous process of gradual transition. Today's concept of urban areas has been built up area that not limited to the center of the town but already urbanized areas including its surrounding suburbs. But at present the center city urbanization areas defined as there is no unified standard. Even draw after peripheral urbanization areas of central cities that are not delimit adjacent parts also is not the contrast obvious in rural areas, but is to make the transition to urbanization and urbanization regions [1-2].

China's rapid urbanization process has become the hotspot of the world's attention, and the rapid urbanization make the country on the whole, cannot provide the service, which is necessary in order to adapt to rapid urbanization and infrastructure, the large influx of population led to urban development and present state of disorder, urban poverty is also growing, the area deprivation phenomenon has become increasingly prominent. We deprive behavior is referred to in area, in the process of the rapid urbanization, the powerful and strong regional based on basic regional and regional spatial position relationship between, with the help of the policy empty and the administrative coercion plundered the resources of the disadvantaged and the vulnerable area, capital, technology, talents, project, ecology, environmental capacity, to pass on all kinds of pollution and so on a series of unfair, the reasonable behavior of the economic and social activities, that is a regional economic development at the expense of the other regional economic development and resources at the expense of the environment [3].

Combined with the related research results and the current situation of economic development, this article has analyzed the urbanization from four aspects the elements of its dynamic mechanism as the following aspects. (1) The changes of the system and the adjustment of the government's public policy have important effects on urbanization. The incremental reform in the system effectively speeds up the transfer of rural surplus labor, promoting the urbanization 
process. Housing marketization reform further stimulates the urban development expand the urban development space, has a driving effect on development of the urbanization. (2) Since entering the new century, our country's economic growth mode has experienced from the consumer to the change of investment, investment promotion and the capital introduction has also become the basic center of the city government's work. (3) Science and technology, education and the other non-economic factors can under the condition of not increasing inputs increase labor productivity, optimizing the economic structure, and the deep and lasting impact on urbanization. Technological progress included not only in the broadest sense of the science and technology and knowledge innovation, including the improvement of system, system of organization structure, the formation of externality and the spillover effect, for the intensive economic growth and urbanization continue to provide the long-term power guarantee for it. Relatively, we demonstrate the modern psychological health development procedures in the figure one.

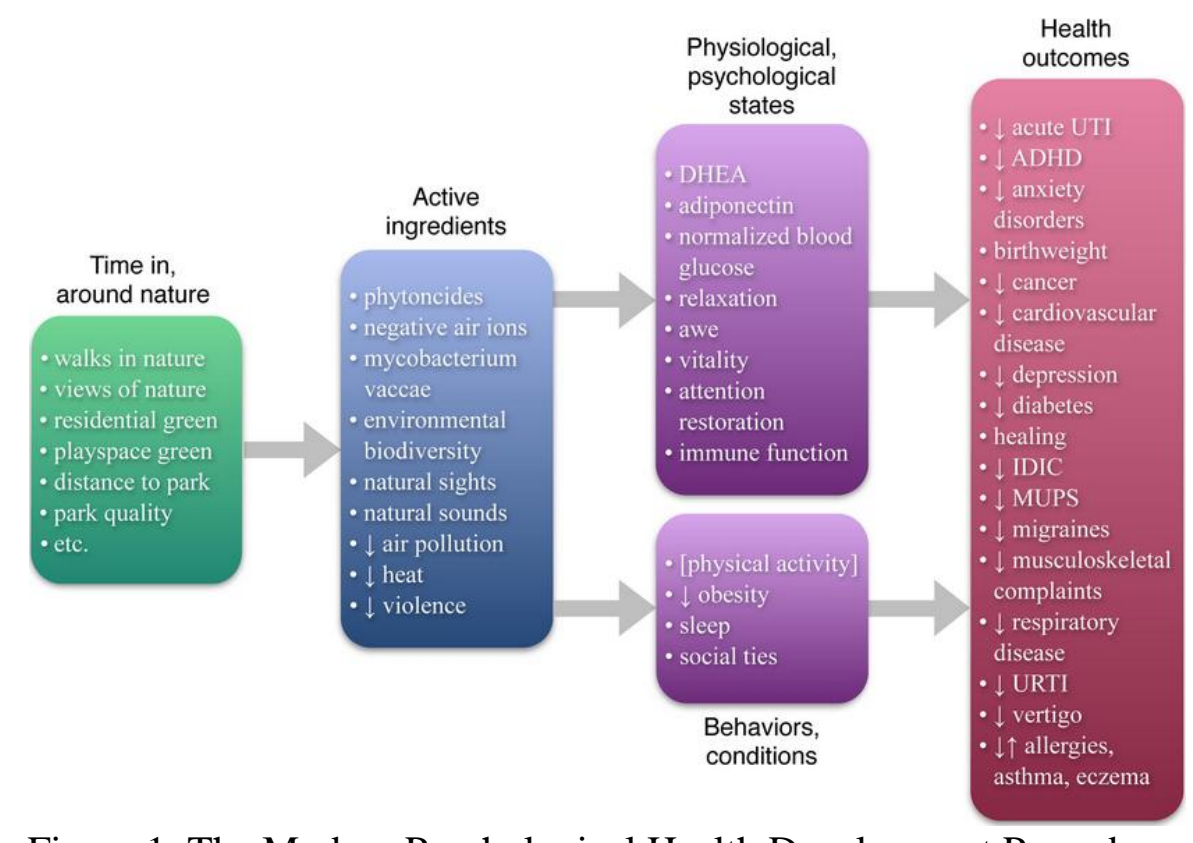

Figure 1. The Modern Psychological Health Development Procedures

In this paper, we conduct research on the modern psychological health problems of the floating population development trend under the urbanization process. Population movements become the present stage our country economy, society, a striking feature of the process of primary demographic transition, long-term stable development of China's future society a huge challenge. Problem defined the social integration of floating population, that is, the process of how to adapt to city life, become the focus of the government, society and academic circles last. In the later sections we will discuss the corresponding issues in detail with in-depth analysis.

\section{The Proposed Methodology}

The Property of the Mental Health. Mental health reform and development in society today, more and more get the attention of people, as standard of mental health has aroused people's attention. Due to the standard of mental health problems is wide and very complex, so far, about the standard of the mental health problem has not been the unified conclusion. But the standard problems on the deep understanding of mental 
health is very necessary, because only in the understanding and grasp the real meaning of mental health and its standard under the premise of to a target is engaged in the education of mental health, preventing the psychological problems of people, and can appear in mental illness after consultation and treatment goals, finally spurred the mental health level [4-5].

From the psychological process, a healthy person's psychological activity is a complete and unified coordination body, this coordination to ensure the individual in the reflection of the objective world in the process of high accuracy and effectiveness. The fact that the knowledge is the starting point of mental health, the act of will is a home to return to the personality features emotion is the intermediary factor between understandings and will. From several aspects of the psychological structure, if they cannot meet regularly to coordinate action, is likely to produce a series of psychological problems or issues. From the individual point of view, each of us has our own long-term stable personality and a person's personality in the absence of obvious severe due to the impact of external factors.

Seen from the relationship of individual and group, each person in the reality can be divided into different groups, different groups is there are differences between the standard of mental health. As for the resilience of basic emotion, the literature reviews have provided us with the suggestions. (1) Whether to model or functional view of emotion regulation, the main research content is according to the regulation of negative emotions, and finishes the emotion regulation as a one-time behavior and Gross of the emotion regulation of two stage process model is put forward, the emotion regulation is considered in the process of sentiment, in the different stages of sentiment that have different emotion regulation. (2) Psychological resilience is individual relatively stable response to negative emotions, resources, and it makes the individual resumed as soon as possible to the normal emotional state, however positive emotions not only reflects the psychological restoring force of the high and low, with the passage of time, the positive emotions by expanding mechanism can improve the individual psychological restoring force that is general process of mutual promotion. (3) The person psychology restoring force is similar, in the face of negative events or circumstances, the individual at the same time of produce negative emotions also can produce with the negative emotions against psychological resilience. Researchers around the psychological resilience of individual emotional, cognitive and physiological effects on that positive emotion are a very important psychological resilience.

The theory from the transverse structure of emotion regulation, the concept of original system of emotion regulation in the deconstruction, through the theoretical discussion and empirical support to rebuild the initial structure of the concept of the emotion regulation, however, due to the structure proposed time is short, still remains to be further revised theoretical and experimental research and verification, in addition, for the specific relationship between each concept or components still need to discuss and more further related research.

The Features of the Urbanization Process. City is the product of the industrial revolution, it is a sign of modern civilization, population, industry and commerce developed, mainly used for residents with non-agricultural population that is the political, economic, cultural, education and information center. Urban centralized embodies the country's comprehensive national strength basic management capacity and international competitiveness, with concentration, scale, organization and the functional effect. Economists normally from the perspective of the relationship between economy and the city, emphasis on urbanization from rural economy to the transformation of urban economy; Geographers emphasized the change of the economic and cultural relations between urban and rural areas, think the city is the center of the 
region on a variety of activities, urbanization is the result of the development of social productivity and cause of agricultural population to the urban population, the form of rural residential areas to the urban settlement form transformation process [6-7].

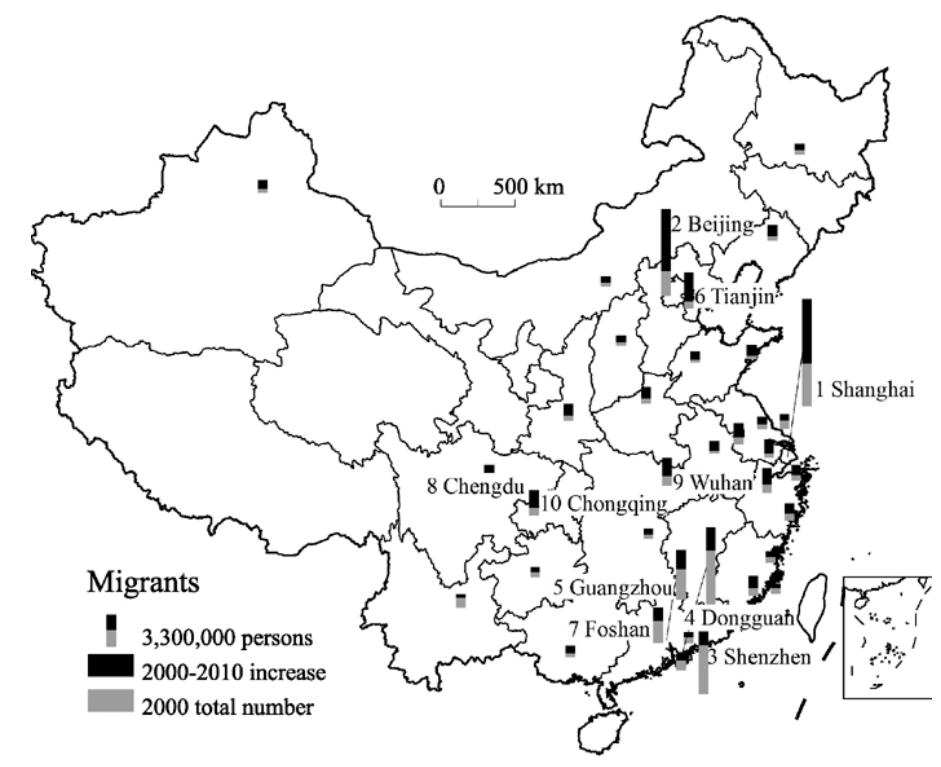

Figure 2. The Urbanization Process and Trend in China

Urbanization is the common law of national economic and social development which is a natural historical process. In general, a country's economic modernization is based on the two processes to complete the industrialization and general urbanization. Industrialization has created modern urban population as the main consumer groups of consumer goods, and industrialization brought about by the social division of labor, can only be realized in the system of urbanization. Industrialization based on urbanization, urbanization is dependent on industrialization. From the perspective of relationship between the economic modernizations in the primary process of creating supply of industrialization, urbanization creates demand. Overall, the above all subject to the understanding of the connotation of urbanization from their respective disciplines and explained, have strong theoretical foundation, but not the comprehensive. Urbanization is essentially a people-centric, affected by many factors which extremely complex and changeable system transformation process, including the hardware structure and software structure of the system of change and general ascension, comprehensive transition from traditional society to modern civilization and the process of change [8].

Perfect in functions and facilities is the city's economic relations, the residents way of life and the human society civilization is widely to rural penetration process. Process of urbanization is more and more peasants off the land free process, also is the rural residents greatly improve the material life and spiritual life, and gradually achieve the coordinated development of urban and rural areas, eventually eliminate the process of urban and rural differences and differences of workers and peasants.

The Floating Population Mental Health. Migration is a complicated process involving a variety of changes. From familiar with living and working environment to a new environment, the floating population will face many problems, such as instability, the lack of social network and social support, language communication barriers with cultural differences and so on these are likely to be an adverse effect on the mental health of the floating population. On immigration has a history of research in the foreign countries, 
research on foreign immigrant mental health were reviewed, for our country to study the mental health of the floating population to provide the certain reference. Immigrants were divided into the initiative, such as shelter, initiative and immigration, such as economic driver of the immigrants, the corresponding countermeasures could be summarized as follows.

- Unit of choose and employ persons should pay attention to humanities concern of the floating population to avoid the discrimination of floating population. Suggested that unit of choose and employ persons of the floating population to carry on the humanistic care, equality and justice to the floating population and floating population, realize the equal pay for equal work, distribution according to work as can enhance the economic income of the floating population on one hand, on the other hand can reduce the discrimination of the floating population of the consciousness, so as to promote their self-esteem, promotion of mental health and happiness.

- To establish the floating population psychological service, provide psychological assistance of the floating population. Floating population vulnerable to a variety of psychological problems, the psychological aid of itinerant population is imperative. Suggestions for each city according to how much set up a certain number of basic floating population family of floating population, here, the floating population can communicate with each other, can through the network video communication with family and also can turn to psychological consultant [9].

- We will improve the social security system for the floating population, to solve the trouble back at home of the floating population. We will improve the medical security system for the floating population, to make them when sick can be timely and effective treatment, medical expenses reimbursement procedures to further simplify, helps reduce the anxiety of the floating population. In addition, to further improve unemployment of the floating population, pension and other social security system, but also to improve the level of their mental health.

In the study of the floating population, push-pull theory model is most widely used theoretical model. Applying this theory model analysis of China's urban and rural population flow as the huge economic driving force is the mainspring of migrant workers to go out. Huge economic difference and income difference between urban and rural areas is the most main reason for the population to the urban mobility. The Chinese model comparing with the international model, while unemployment in the countryside, the city policy tilt and the influence factors of flow distance, there are some things in common, however, the international rural violence, the rural population has no control on growth, displaced by factors such as the farmers lost their land in China's model of push-pull find similarities.

\section{Conclusion}

In this paper, we conduct research on modern psychological health problems of floating population development trend under the urbanization process. At present, the research on mental health object diversity, methods, the characteristics of diversification, contents enrichment, the neural mechanism of psychological barriers, psychosomatic interaction mechanism study, drugs and the mechanism of Internet addiction research, the mechanism research of the mental health service system become a hotspot. From the point of the situation of the national mental health research, 
the research mainly includes only primary children mental health problems, psychological health problems of students, psychological health problems of special groups, professional group of mental health problems and mental health problems of five aspects of Internet population. Our research focuses on the research on the psychological health problems of floating population development trend that serves as the basic research on the corresponding areas.

\section{References}

[1] Gruber, June, et al. "Happiness is best kept stable: Positive emotion variability is associated with poorer psychological health." Emotion 13.1 (2013): 1.

[2] Human, Lauren J., et al. "Is change bad? Personality change is associated with poorer psychological health and greater metabolic syndrome in midlife." Journal of personality 81.3 (2013): 249-260.

[3] Routledge, Clay, et al. "Nostalgia as a resource for psychological health and well being." Social and Personality Psychology Compass 7.11 (2013): 808-818.

[4] Benish-Weisman, Maya, et al. "Healing stories: Narrative characteristics in cancer survivorship narratives and psychological health among hematopoietic stem cell transplant survivors." Palliative and Supportive Care 12.04 (2014): 261-267.

[5] Campos, Belinda, et al. "Familism and psychological health: the intervening role of closeness and social support." Cultural Diversity and Ethnic Minority Psychology 20.2 (2014): 191.

[6] Chambers, Suzanne Kathleen, et al. "Intervening to improve psychological outcomes after cancer: What is known and where next?." Australian Psychologist 49.2 (2014): 96-103.

[7] Segovia, Francine, et al. "Optimism predicts positive health in repatriated prisoners of war." Psychological Trauma: Theory, Research, Practice, and Policy 7.3 (2015): 222.

[8] Mastura, Johar, et al. "Effect of low-impact aerobic dance exercise on psychological health (stress) among sedentary women in Malaysia." (2012).

[9] Bartholomew, Kimberley Jane, et al. "Job pressure and ill-health in physical education teachers: The mediating role of psychological need thwarting." Teaching and Teacher Education 37 (2014). 